\title{
Design of SCM-based System Software for Packing Machine
}

\author{
Yuan Qinghui ${ }^{1, a,{ }^{*}}$, Nie Xiujun ${ }^{1, b}$ and Feng Guofang ${ }^{2, c}$ \\ ${ }^{1}$ Binzhou Polytechnic, Binzhou, Shandong Province China,256603 \\ ${ }^{2}$ Sixth Primary Schools in Bincheng, Binzhou, Shandong Province China,256600 \\ asdyqh1979@126.com, b443183538@qq.com, ${ }^{\mathrm{c}} 48908892 @ q q . c o m$
}

\begin{abstract}
This paper introduces a design of packing machine system based on SCM, the system uses SCM as the main control chip and data processing, writting language programming with the Keilu Vision MCU software, debugging, and using the software to import hex file with protues for software and hardware FBI.
\end{abstract}

Keywords: SCM, Packing machine, Keil C51

\section{Introduction}

With the development of society and the development of industry, the automation production line is more and more widely used in various production factories. The emergence of the packing machine is better adapted to the development of society, which not only makes the products contain higher scientific and technological content, but also improves the production efficiency of the product. The so-called packing machine puts a certain amount of the finished product into the packaging box through the conveyor belt, and it sends off the product through the box conveyor belt. Through the use of such a device, we can greatly improve the production efficiency of enterprises and reduce the labor costs of enterprises.

\section{Packing machine system}

In this paper, the AT89C51 microcontroller based control chip combined with peripheral circuits constitute a multi function automatic packing machine system. The packing machine system is mainly composed of the following modules: data processing and control module SCM AT89C51, $4 * 4$ matrix keyboard input module, ISD2560 voice chip module (including a microphone and speakers), digital tube display module, sensor module and other ancillary module (including the buzzer, status indicator and reset circuit).

Firstly, the hardware circuit diagram is designed in the Protues hardware simulation software environment. Then, the software programming of the system is carried out in the Keil software environment, which is compiled and debugged, and the hex file is generated. This hex file is the source code for the implementation of the hardware circuit.We load the generated hex file to the AT89C51 microcontroller chip, and then run the hardware circuit in the software environment, so the packing machine system can be normal operation ${ }^{1}$. 


\section{The system software design of the packing machine}

(1) System software design program

The software program design of the packing machine system mainly includes: initialization program, key detection program, key processing subroutine, external testing program, voice broadcast program.

(2) System function software implementation

Initialization procedure

When the container system is opened, the system should be initialized first, including the two motor and the status of the lights, LED digital tube display initialization and so on.In the initialization process, the system for some of the data set fixed temporary storage unit, among them, $40 \mathrm{H}$ and $41 \mathrm{H}$ are set to the product storage unit, $42 \mathrm{H}$ and $43 \mathrm{H}$ are set to the product box storage unit, $44 \mathrm{H}, 45 \mathrm{H}$ and $46 \mathrm{H}, 47 \mathrm{H}$ are respectively set to the current product and the product box storage unit.

The initialization list is as follows:

ORG $\quad 0000 \mathrm{H}$

AJMP MAIN

ORG $\quad 0033 \mathrm{H}$

MAIN:CLR P1.0 ; Turn off the two belt

CLR P1.1

SETB P1.2 ;Turn off the sound light alarm and show the green light

CLR P1.3

CLR P1.4

MOV R0, \#40H ; First, the display number is set to zero, the storage unit is $40 \mathrm{H}-47 \mathrm{H}$

MOV R2, \#08H

NEXT1: MOV A,\#00h

MOV@r0,A

INC R0

DJNZ R2，NEXT1

ACALL DISP ; Call display program

1. RET

Key detection program

After the completion of the initialization of the system, the system will enter the state of the keyboard scanning, After the detection of a button is pressed, the system will automatically turn to the corresponding key processing subroutine.

Keyboard scanner

SAOMIAO: MOV P2, \#0FFH ; First, the P2 port is set to high level

CLR P2.4 ; Set the P2.4 to a low level

JNB P2.3,L1 ;If the P2.3 port is low, call delay procedure

AJMP D2 ;If the P2.3 port is not low, then continue to detect the next I/O port

L1: ACALL DLY-S $\quad$;Call delay procedures, remove the keyboard jitter JNB P2.3, KEY1; ;f P2.3 is still low, then Turn to the corresponding keyboard processing program

D2: JNB P2.2,L2 ; 
AJMP D3

L2: ACALL DLY-S JNB P2.2,KEY2

D3: JNB P2.1,L3

AJMP D4

L3: ACALL DLY-S JNB P2.1,KEY3

D4: JNB P2.0,L4 AJMP D5

L4: ACALL DLY-S JNB P2.0,KEY4

D5: SETB P2.4 ; Set the P2.5 port to a low level

CLR P2.5

NB P2.3,L5

AJMP D6

L5: ACALL DLY-S

JNB P2.3, KEY5

D6: JNB P2.2,L6

AJMP D7

L6: ACALL DLY-S

NB P2.2,KEY6

D7: JNB P2.1,L7

AJMP D8

L7: ACALL DLY-S

JNB P2.1,KEY7

D8: JNB P2.0,L8

AJMP D9

L8: ACALL DLY-S

JNB P2.0,KEY8

D9: SETB P2.5 ; Set the P2.6 to a low level

CLR P2.6

JNB P2.0,L9

AJMP D10

L9: ACALL DLY-S

JNB P2.0,YOU

D10: JNB P2.1,L10

AJMP D11

L10: ACALL DLY-S

NB P2.1,ZUO

D11: JNB P2.3,L11

AJMP D12

L11: ACALL DLY-S

JNB P2.3,KEY9

D12: JNB P2.2,L12

AJMP D13 
L12: ACALL DLY-S

JNB P2.2,KEY0

D13: SETB P2.6

CLR P2.7

JNB P2.1,L13

AJMP D14

L13: ACALL DLY-S

JNB P2.1,INPUT

D14: JNB P2.2,L14

AJMP SAOMIAO

\section{L14: ACALL DLY-S}

JNB P2.2,ESC

AJMP SAOMIAO

Key processing subroutine

When the keyboard is detected, the system of the packing machine can automatically turn to the key processing program. This part is the main part of the system, and all kinds of behavior of the system are based on the results of this part. In this part, the system will judge the information input by the staff, in order to achieve the corresponding function.

External testing procedure

External test program is used to detect the conveyor belt on the product and the product box and sends the data to the microcontroller. After the data is processed by the single-chip microcomputer, the information of the control transmission belt is sent out.Detection of products and products box are completed by the external infrared tube,and infrared tube of detection product box is connected to the microcontroller p3.2 port (external interrupt 0 ), and infrared tube of detection product is connected to the MCU p3.3 port (external interrupts 1).Any one of Keil C51 sends out the signal, SCM will interrupt occurs, and the corresponding data plus 1 , then which is compared with the parameter set. we can determine conveyor belt of the state according to the results of the comparison, or let the whole system into the state to stop the waiting.

Voice broadcast program

This design uses the ISD2560 voice chip and achieves recording and playback function with AT89C51 single-chip control of voice chip.

Recording subroutine

When recording, we press the recording key, and SCM sets the starting address of the voice segment through the I/O port line, and then makes the PD, P/R and /CE terminal for the low level to start recording; At the end, we loose the button, and SCM makes the /CE side to return to the high level, that is, we have completed the recording of a voice. With the same method we can continue to record second, third, and so on. It should be noted that the recording time can not be more than the time set in advance of each voice 2 。

Playback program

When playing, according to the voice content to be broadcast, we can find the starting address of the corresponding speech segments and sent through the mouth line.Then the $\mathrm{P} / \mathrm{R}$ is set to high level ,and PD is set to a low level.That let the /CE end generate a negative pulse to start playback, and at this time SCM just waitting for end signal of ISD2560 information, that is the generation of /EOM. Signal is a negative pulse, and in the negative pulse rising 
edge the period of voice play end. So SCM must detect /EOM rising along in order to play the second paragraph, or Playing voice is not continuous and pops, that we must pay attention to this point in the preparation of software ${ }^{3}$.

\section{System debugging}

After the system hardware circuit design and software program design are completed, first of all, in the Proteus circuit simulation environment, by editing, compiling, assembly, connection, the generated.HEX file is loaded into the MCU AT89C51, then we debug the whole system. The following is the main problems encountered in the debugging process and the corresponding solutions:

(1) Problem description: In the display module, the design of the initial program is the use of dynamic scanning display, however, in the process of running the interval there will be out of the state and the need for more I/O port, which can not meet the needs of other functions.

Cause analysis and solution: Because of this dynamic scanning does not have the data latch function, so it is necessary to scan the data from time to time. But because the system needs to complete more functions, there will have a longer interval between the each two times scanning, which will appear in the state of extinction, and dynamic scanning need to take up $16 \mathrm{I} / \mathrm{O}$ port, 8 address selection and 8 code delivery, so there is a big waste for I/O port.Later we used serial port display, each digital tube plus a 74HC164 chip before the digital control. The chip is not only able to shift, but also can give the serial data into parallel data, what's more, which has a latch function and do not need to scan all the time. In this way, there only requires RXD and TXD two ports and saves a large number of I/O resources.

(2) Problem description:When the data is modified and the left and right shifts, the continuous shift can not be carried out, otherwise the unit will be dark and unable to modify the data.

Cause analysis and solution:This article is a procedural problem. Because when the shift is carried out, the unit passed by the unit has been decoded into FFH, and due to the low level, which is the state of extinction in the digital tube. The original procedure is as follows:

ZUO: $\mathrm{MOV}$ A,R1

CJNE A,\#47H,NEXT2

MOV R1,\#40H

MOV@R1,\#10

ACALL DISP

ACALL DELAY

AJMP SAOMIAO

NEXT2: INC R1

MOV@@1,\#10

ACALL DISP

ACALL DELAY

AJMP SAOMIAO
;Left shift function key procedures, hold down the key, you can change

; Need to modify the number of new units or need to modify the unit 
In order to avoid this problem, the program is changed to the following:

\begin{tabular}{cl} 
ZUO: & \multicolumn{1}{c}{ A,37H } \\
MOV & @1, A \\
MOV & A,R1 \\
MOV & R1,\#40H \\
MOV & $@ R 1, \# 10$ \\
ACALL DISP \\
ACALL DELAY \\
AJMP & SAOMIAO \\
NEXT2: INC $\quad$ R1 \\
MOV $@$ QR1,\#10 \\
ACALL DISP \\
ACALL DELAY \\
AJMP & SAOMIAO
\end{tabular}

At each input data, the same data is sent to the $37 \mathrm{H}$ unit. When the left and right shift, there need not send the data to modify the data, so you can avoid the above situation.

Problem description: In the continuous left and right shift, there will be the case that data moves several units at a time, which makes it unable to modify the data in the unit that needs to be modified.

Cause analysis and solution: It occurs because the staff is unable to achieve the timely to let go when a key is pressed, which will make it remain a few tens of milliseconds. During this period, the shift program will be carried out many times, and thus the above situation appears. This can be avoided by adding a delay time to the left and right shift program.

\section{Conclusion}

In this paper, we design a kind of automatic packing machine system with AT89C51 MCU as the main control chip combined with peripheral circuit.The system can automatically complete the packaging of the finished product, and can carry out manual intervention to achieve the purpose of artificial control when the system is wrong or we think it is necessary. The whole system in the man-machine exchange is still relatively complete, including voice, sound and light, etc., which can better let the staff to operate the entire system.

\section{References}

[1] Sun Yucai, MCS-51 series single chip microcomputer and its application edition. Nanjing: Southeast University press, 1997, p. 1〜19.

[2] Liu Lianji.ISD voice chip recording / release device and circuit design. Journal of Qingdao Ocean University, Vol. 3 (1997), p. 291-299.

[3] Xiao Hairong, Wang Fengying, Yang Jinqing, etc., the design of [J]. recording system AT89C2051 and ISD2560 micro computer based information, Vol. 1 (2004), p. 63-64. 